Design Prospective cohort.

Setting Calgary, Canada over five ice hockey seasons (2013/ 14-2017/18).

Participants Male and female youth ice hockey players ages 11-18.

Assessment of Risk Factors Participant baseline reports of mouthguard use (yes/always and sometimes use, no/never use), helmet age (newer $/<2$ years old, older $/ \geq 2$ years old), and other important covariables [i.e., weight (kilograms), age group (under-13, under-15, under-18), position (forward, defense, goalie), concussion history (yes, no), body checking policy (allowed, disallowed)] were collected near the start of each season. Moreover, each player's participation hours were collected throughout each season.

Main Outcome Measurements Number of medically diagnosed or therapist identified suspected concussions using validated surveillance methodology in games and practices over 5 seasons of play.

Results Multilevel negative binomial regression adjusted for player position, level of play, body checking policy, concussion history, weight, cluster by team, and offset by playerhours was used. The model included 426 concussions suffered by 369 players (from 394 player-seasons; 29 players had recurrent concussions in a single season) over 4,541 player-seasons (271,148.7 player hours). The model demonstrated that players who reported wearing a mouthguard had a $28 \%$ lower rate of concussion compared to those who did not $(\mathrm{IRR}=0.72$, 95\%CI: 0.55-0.93). Moreover, there were no differences in the concussion rate between newer and older helmet ages (IRR $=0.94$, 95\%CI: $0.76-$ 1.16).

Conclusions Protective equipment is an important consideration for concussion prevention and player safety. Wearing a mouthguard was associated with a lower concussion rate and policy mandating mouthguard use should be considered in youth ice hockey. More specific helmet age categories may require further investigation.

\section{FUNCTIONAL MOUTHGUARD DESIGN TO ENHANCE THE PROTECTIVE CAPABILITY AND ATHLETE COMFORT}

\footnotetext{
${ }^{1,2}$ Naser Nasrollahzadeh, ${ }^{2}$ Dominique P Pioletti, ${ }^{1}$ Martin Broome. ${ }^{1}$ Division of oral and maxillofacial surgery, Lausanne University Hospital (CHUV), Lausanne, Switzerland; ${ }^{2}$ Laboratory of Biomechanical Orthopedics, Institute of Mechanical Engineering, EPFL, Lausanne, Switzerland
}

10.1136/bjsports-2021-IOC.396

Introduction Athletes of contact sports are prone to cranial, orofacial and dental injuries in case of a traumatic head impact. Mouthguards can be potentially beneficial in reducing the injury risk by changing the dynamics of an impact to teeth. Indeed, dissipative capacity of mouthguard materials influences the extent of transferred force. However, the effect of geometrical/structural attributes should not be neglected on the mouthguards performance.

Objective The aim of this study is to evaluate the role of different design variables in protective capability of mouthguards and to find an optimal configuration by means of computer aided engineering.

Design and Setting A detail anatomical human upper jaw model was developed including teeth, periodontal ligament and maxilla bone. The incisor impact with an ice hockey puck was then simulated by finite element analysis with and without mouthguard. Various mouthguard configurations were designed by employing different material properties, laminated composite arrangement, layers thickness, and space inclusion.

Main Outcome Measurements The maximum effective stress on the incisors, contact force profile and stress distribution were compared to evaluate effect of different parameters on mouthguard protective performance.

Results While larger thickness always reduces the risk of injury in all configurations, the effectiveness of space inclusion and composite layers arrangement are design dependent. The optimal configuration was obtained when we combined graded stiffness layers (2000 to $20 \mathrm{Mpa}$ ) arrangement with a predefined gap $(1 \mathrm{~mm})$ in front of incisors. In this specific design, limiting the mouthguard thickness to $3 \mathrm{~mm}$ resulted in acceptable protective performance compared to $4 \mathrm{~mm}$ case and was preferred for the sake of athletes comfort.

Conclusion Both structural and material properties are playing a key role in shock absorbing capabilities of mouthguard. There is a need to practice multi-materials $3 \mathrm{D}$ printing for fabrication of customized mouthguards to maximize their performance and comfort.

\section{COVID-19 AND SPORTS - AN ONLINE SURVEY ON THE IMPACT OF THE PANDEMIC AND POSSIBLE PREVENTIVE MEASURES}

${ }^{1}$ Friedemann Schneider, ${ }^{1,6}$ Armin Runer, ${ }^{2}$ Francesco Burkert, ${ }^{3}$ Jesse Seilern Und Aspang, ${ }^{4}$ Holm Schneider, ${ }^{5}$ Elena Pocecco. 'Department of Orthopaedics and Traumatology, Medical University of Innsbruck, Innsbruck, Austria; ${ }^{2}$ Department of Internal Medicine II, Infectious Diseases, Pneumology, Rheumatology, Medical University of Innsbruck, Innsbruck, Austria; ${ }^{3}$ Medical University of Vienna, Vienna, Austria; ${ }^{4}$ Department of Pediatrics, University Hospital Erlangen, Erlangen, Germany; ${ }^{5}$ Department of Sports Science, Medical Section, University of Innsbruck, Innsbruck, Austria; ${ }^{6}$ Department for Orthopaedic Sports Medicine, Technical University Munich, Munich, Germany

\subsection{6/bjsports-2021-IOC.397}

Background The COVID-19 pandemic led to the implementation of worldwide governmental restrictions and preventative measures with large impact on social life.

Objective To investigate the effects of the pandemic on individual and general sport activities in an effort to provide information for safe return to community sports.

Design An electronic survey was launched in June 2020 in German and English language. The anonymous questionnaire collected epidemiological data and responses 'before','during' and 'after' confinement conditions. Descriptive statistics and logistic regression analysis were used.

Setting Most participants practiced their sport in Europe (93.9\%); 68.5\% were active athletes, $10.1 \%$ coaches, $10.1 \%$ had other sports functions, $11.3 \%$ indicated no regular sports activity.

Participants 1336 adults (30.5 \pm 11.7 years; $54.0 \%$ women) participated in the survey.

Assessment of Risk Factors Risk factors for a serious course of COVID-19 disease were queried.

Main Outcome Measurements The type, extent and intensity of physical activity were defined as main outcome measures.

Results During confinement, $15.7 \%$ could perform their main sport unrestricted, $43.5 \%$ stated a reduced amount of time spent on sporting activities, $46.4 \%$ a reduced intensity level. Most participants were neither aware of screening measures 